\title{
The Mass-Mobility Correlation Redux: The Conformational Landscape of Anhydrous Biomolecules
}

\author{
John A. McLean \\ Department of Chemistry, Vanderbilt Institute of Chemical Biology, Vanderbilt Institute for Integrative \\ Biosystems Research and Education, Vanderbilt University, Nashville, Tennessee, USA
}

\begin{abstract}
Structural separations on the basis of gas-phase ion mobility-mass spectrometry are increasingly used for the analysis of complex biological samples. As a tool to elucidate biomolecular structure, ion mobility-mass spectrometry methods are unique in that direct molecular structural information is obtained for all resolved species, largely irrespective of the complexity of the sample. Computational approaches are used to interpret and discern structural details consistent with the empirical results. To a first approximation, correlations of mobility with mass allow for qualitative identification of the molecular class to which a particular species belongs. These correlations allow simultaneous characterization of different classes of biomolecules, which provides a means for combining omics measurements, such as lipidomics, proteomics, glycomics, and metabolomics, in the same analysis. Examination of the correlation of fine structure reveals that specific structural motifs, chemical functionality, chemical connectivity, and composition may also be determined, depending on the specific biomolecular class. Mapping the coarse and fine structure in ion mobility-mass spectrometry conformation space measurements provides an atlas for interpretation and discovery in complicated spectra. (J Am Soc Mass Spectrom 2009, 20, 1775-1781) (c) 2009 American Society for Mass Spectrometry
\end{abstract}

$\mathrm{G}$ as-phase separations on the basis of migration and diffusion of ions through a neutral gas under the influence of an applied field have existed for over a century. The first examples of gasphase ion mobility (IM) include the quantitative studies of ionized gases by Rutherford shortly after the discovery of X-rays [1, 2]. To place these early IM experiments in the context of mass spectrometry (MS), it would be almost another decade before J. J. Thomson would construct his first mass spectrograph [3]. Over the historic span of IMS development, the technique has been referred to by several names, including plasma chromatography [4], ion chromatography [5], and the currently accepted term ion mobility spectrometry (IMS). A detailed account on the historic development of IMS and milestones in the progress of gas-phase IM techniques is described elsewhere [6, 7]. For many years, IMS separations were used for the fundamental study of atomic and small molecular ions in plasma physics. In the 1960s and 70s, a transition occurred from using IMS primarily as a fundamental physics research tool to using it as a separations device for analytical and physical chemistry applications. Notably, the early 1960s also marked the first reports of combining IMS with MS (IM-MS) [8, 9].

Address reprint requests to Professor J. A. McLean, Department of Chemistry, Vanderbilt University, 7330 Stevenson Center Nashville, TN 37215, USA. E-mail: john.a.mclean@vanderbilt.edu
On the basis of these early IM-MS instruments, it was proposed that with proper calibration, IMS instruments could be operated as atmospheric pressure mass spectrometers in that mass could be assigned to a particular IM drift time [10, 11]. This supposition was derived from plotting curves of mass as a function of mobility, which resulted in highly correlated calibration curves. However, this notion was qualified by Horning and colleagues in that highly correlated mass-mobility relationships are only valid for structurally-related compounds, and concluded that "identification of the ion species based solely upon mobility data is at best speculative and should be discouraged [12]." Subsequently, a rigorous treatment derived from the kinetic theory of gases, reviewed the interdependencies of the physical parameters of separation on the measured mobility for drift tube IM [13-15], which to a first approximation, given appropriate approximations, results in the following:

$$
K_{0}=\frac{(18 \pi)^{1 / 2}}{16} \frac{z e}{\left(k_{B} T\right)^{1 / 2}}\left[\frac{1}{m_{i}}+\frac{1}{m_{n}}\right]^{1 / 2} \frac{760}{p} \frac{T}{273} \frac{1}{N_{0}} \frac{1}{\Omega}
$$

where these parameters include the reduced mobility ( $K_{0}$, or mobility normalized to STP conditions), charge of the ion $(z e)$, Boltzmann's constant $\left(k_{B}\right)$, temperature $(T)$, the reduced mass of the ion-neutral collision pair (ion and neutral masses of $m_{i}$ and $m_{n}$, respectively), pressure $(P)$, number density of the drift gas at STP $\left(N_{0}\right.$, 
$\left.2.69 \times 10^{19} \mathrm{~cm}^{-3}\right)$, and the ion-neutral collision cross section $(\Omega)$. Because mobility $(K)$ is defined as the proportionality constant relating drift velocity $\left(v_{d}\right)$ in a gas under the influence of an electrostatic-field $(E)$, i.e., $v_{d}=K E$, by substitution and rearrangement eq 1 is typically rewritten to solve for the ion-neutral collision cross section:

$$
\Omega=\frac{(18 \pi)^{1 / 2}}{16} \frac{z e}{\left(k_{B} T\right)^{1 / 2}}\left[\frac{1}{m_{i}}+\frac{1}{m_{n}}\right]^{1 / 2} \frac{t_{d} E 760}{L} \frac{T}{p} \frac{1}{273} N_{0}
$$

Thus, by measuring the drift time $\left(t_{d}\right)$ of an ion population across a drift tube of known length $(L)$, the ion-neutral collision cross section can be determined. Often 2D IM-MS data are reported as the arrival time distribution (ATD, corresponding to $t_{d}+$ time the ion resides in regions outside of the drift cell) versus $\mathrm{m} / \mathrm{z}$, rather than collision cross section versus $\mathrm{m} / \mathrm{z}$, because of the nonlinear transformation of drift time-to-collision cross section with mass. The significance of these relationships is that the ion-neutral collision cross section corresponds to the orientationally averaged surface area of the ion, which yields direct structural information.

Based on the fundamental underpinnings of IM theory, a particularly important milestone in the development of IM-MS for biological applications occurred in the mid-1990s when Bowers and colleagues first reported IM-MS structural studies for peptides [16, 17], and for structural analysis of intact proteins by Jarrold, Clemmer, and coworkers [18, 19]. More recently, Robinson and coworkers have extended IM-MS strategies to the elucidation of protein complex quaternary structure [20-23]. To assign potential structural components to the systems investigated, computational approaches and chemical intuition are used to interpret structures consistent with those that are measured. In large part, this work and that of others in the IMS and IM-MS fields denotes a period of rapid and expanding work over the past decade, resulting in the development of biological IM-MS as a multidimensional separations tool for identifying species in complex biological samples and for characterizing biomolecular structure. Importantly, the strength of IM-MS techniques in life sciences research is in utilizing the unique information afforded by integrating the information inherent in both the IM and the MS separation dimensions.

As a biomolecular structural analysis tool, it is important to place these relatively new capabilities in context with more widely accepted methods. As an example for structural proteomics, Figure 1 compares current experimental and theoretical methods for the determination of structural elements at different levels (e.g., protein secondary, tertiary, and quaternary structure), the utility of methods for the analysis of complex samples, and the ability to provide direct or indirect structural determinations. A description of these techniques can be found in several recent reviews [24, 25]. The presented array of techniques is merely a sampling of the major methods that are currently used in their typical manner and is not intended to be comprehensive, which is beyond the scope of this report. Note that the level of relative information obtained between dif-

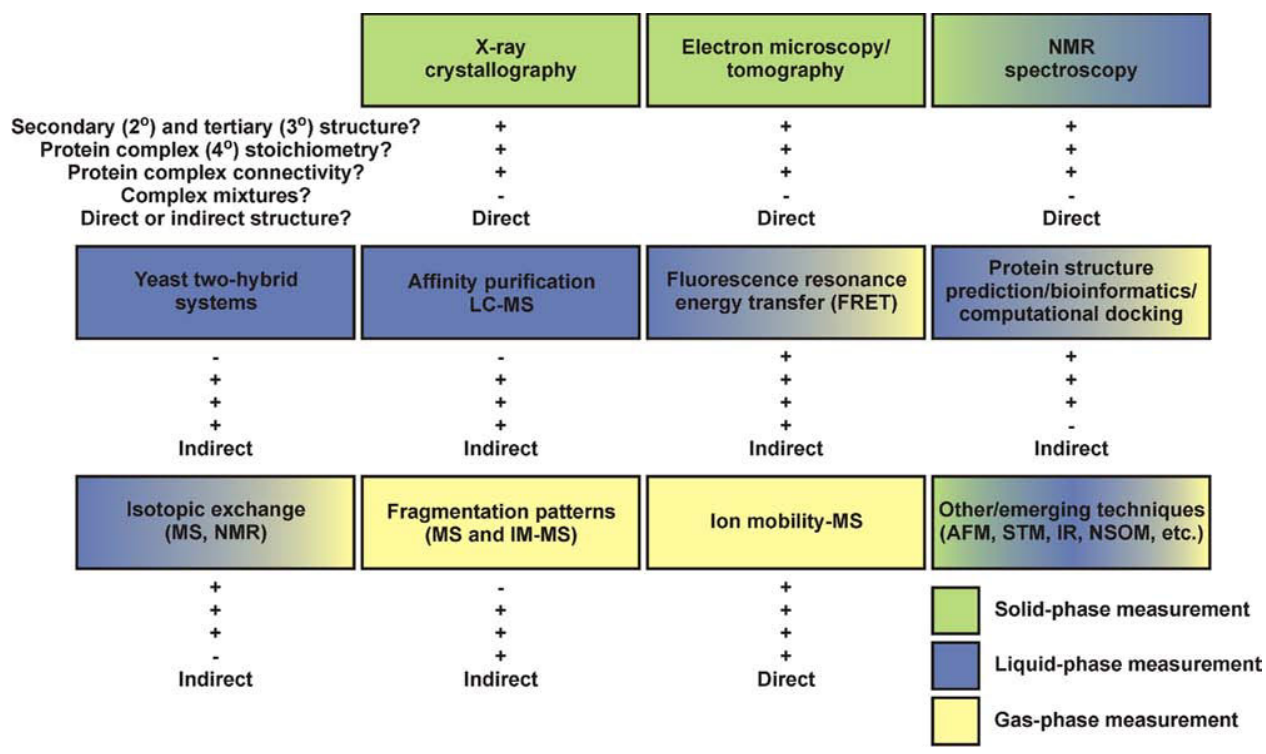

Figure 1. A listing of contemporary experimental and theoretical methods for the determination of peptide, protein, and protein complex structure. The coloring of each panel indicates whether the technique is typically used for solid, liquid, or gas-phase measurements by green, blue, and yellow, respectively. Below each panel is the type of information commonly provided, if it is commonly used for complex samples, and if the structural information obtained is directly or indirectly determined. These designations are for how the different methodologies are typically used, rather than including all possible selected cases. 
ferent techniques may be rather varied and the use of multiple techniques in tandem can further enhance the capabilities listed. A significant advantage of IM-MS approaches to determining biomolecular structure is that inherent in the $2 \mathrm{D}$ dataset is direct structural information for all of the analytes that are present, largely irrespective of the complexity of the sample. Furthermore, modern embodiments of IM-MS typically limit ion transmission efficiency losses and provide limits of detection similar to that of MS-only instruments, while structural information is extractable from trace amounts of sample. Complementary to atomic resolution structural determinations, such as those afforded by X-ray crystallography and NMR spectroscopy, structural information in IM-MS is interpreted by the combination of computational approaches to yield structural information consistent with the empiricallyderived collision cross section data. Depending on the structural hypothesis being queried, oftentimes this is sufficient, given the additional benefits of IM-MS to answer questions yielding important chemical biological information. Aside from discerning structural details of purified samples, the low-resolution structural separations that are afforded provide unique opportunities in the analysis of complex biological samples.

The present report is intended to provide a framework for interpreting IM-MS conformation space, both in a coarse-grained approach and also in the correlation fine structural details that yield qualitative information that can be mined from IM-MS data. There are numerous reports for selected systems describing subtle (or not so subtle) differences in the mobilities or collision cross sections that are obtained for structurally-related biomolecules. However, these differences have largely not been described as a means for mapping conformation space in IM-MS measurements [26, 27]. In the analysis of complex biological materials, such an atlas of conformation space is a prerequisite for integrating various omics measurements to a common IM-MS platform without the commensurate need for extensive sample purification and sample preparation. This report first describes what is obtainable from a coarse-grained examination of IM-MS data in bioanalysis and is followed by a survey of fine structure characterization within molecular classes to enhance molecular characterization in IM-MS measurements.

\section{Structural Separations in Conformation Space-A Coarse-Grained Examination}

The combination of IM-MS provides both ion structural information on the basis of ion-neutral collision cross section (or apparent surface area of the ion) and massto-charge $(\mathrm{m} / \mathrm{z})$. In contrast with highly orthogonal multidimensional separations [28], in IM-MS the two separation dimensions are correlated, which can be simultaneously a challenge and a significant advantage. Fundamentally, this correlation can be rationalized by dimensional analysis. Given the relatively few types of atoms involved in the composition of most biomolecules $(\mathrm{C}, \mathrm{H}, \mathrm{O}, \mathrm{N}, \mathrm{P}$, and $\mathrm{S})$, the mass and volume of a molecule are largely related by a narrow range of density, i.e., biomolecular mass scales as volume, or length cubed, while IM collision cross section, or surface area, scales as length squared. Thus, the average density of different types of biomolecules is largely dictated by (1) the type of monomer units comprising the molecule (e.g., amino acids in proteins, nucleotides in nucleic acids, and sugars in polysaccharides), (2) the polymeric organization of the monomer units (e.g., linearly in proteins and nucleic acids and branched in polysaccharides), and (3) the prevailing intramolecular folding forces of the molecule. Because the physical characteristic giving rise to separation selectivity is structure, the 2D separation space in IM-MS is termed conformation space [29].

In the analysis of complex biological samples, such as those expected to contain analytes encompassing multiple molecular classes, the average densities of different classes differ in the order oligonucleotides $>$ carbohydrates $>$ peptides $>$ lipids [26, 27, 29-31]. Conceptually, this gives rise to correlated mobilitymass relationships, each possessing a different slope for each molecular class as illustrated hypothetically in Figure 2a. Thus, at a given $\mathrm{m} / \mathrm{z}$, the characteristic drift time for different types of molecules vary in the order lipids $>$ peptides $>$ carbohydrates $>$ oligonucleotides and with predictable drift times for specific separation conditions. A plot of collision cross section versus $\mathrm{m} / \mathrm{z}$ for a large number of singly-charged biologically-relevant lipids $(n=53)$, peptides $(n=610)$, carbohydrates (191), and oligonucleotides $(n=110)$ is shown in Figure $2 b$, for species in the mass range $<2000 \mathrm{~m} / \mathrm{z}$. Although these signals are plotted as collision cross sections rather than drift times, to normalize the data for experimental conditions and because of the nonlinear transformation as discussed, the separation of molecular classes is even more prominent when signals are projected as drift times. For example, Figures $2 \mathrm{c}$ and d illustrate baseline separation of lipids and peptides using MALDI-IM-MS in the molecular profiling of thin tissue section of a human glioblastoma and of peptides and carbohydrates in the analysis of the model glycoprotein ribonuclease b. These examples illustrate that qualitative assignment to molecular class in conformation space and identification on the basis of $\mathrm{m} / \mathrm{z}$ could yield a rapid means for combining omics measurements, such as combined lipidomics and proteomics in Figure 2c, and combined proteomics and glycomics in Figure 2d. It is important to note that the correlations depicted in Figure 2 are for positive mode ionization. While for some compound classes negative mode ionization is preferred, there are relatively few reports providing negative ion collision cross section data. Thus, in the negative mode these correlations may be strikingly different and of utility in their own right. 

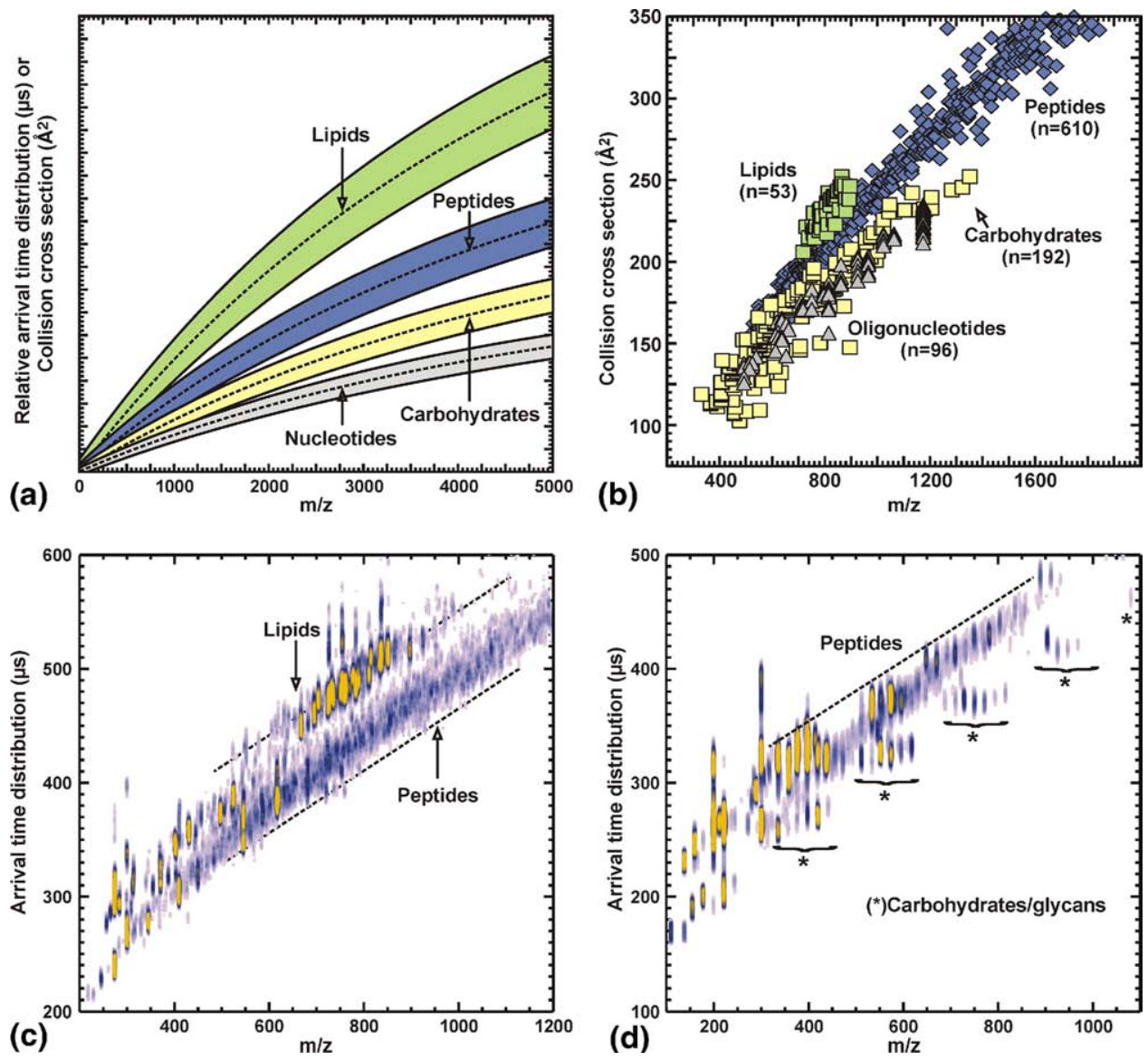

Figure 2. (a) A hypothetical depiction of where singly-charged analytes (e.g., produced by MALDI) of different molecular class are observed in IM-MS conformation space. (b) A plot of collision cross section as a function of $\mathrm{m} / \mathrm{z}$ for different biologically relevant molecular classes, including oligonucleotides $(n=96)$, carbohydrates $(n=192)$, peptides $(n=610)$, and lipids $(n=53)$. All species correspond to singly charged ions generated by using MALDI, where error $\pm 1 \sigma$ is generally within the data point. Values for peptides species are from L. Tao et al., J. Am. Soc. Mass Spectrom. 2007, 8, 1232-1238. (c) A 2D plot of MALDI-IM-MS conformation space for the simultaneous analysis of lipids and peptides directly from a thin tissue section $(12 \mu \mathrm{m})$ of a fresh frozen human glioblastoma. (d) A 2D plot of MALDI-IM-MS conformation space for the simultaneous analysis of peptides, glycans, and in-source decay carbohydrate fragments obtained from proteolysis (trypsin) and N-linked glycan release (PNGase F) of the glycoprotein ribonuclease b. Species corresponding to carbohydrates are indicated by asterisks. Dashed lines are to assist visualization of the different classes of molecules. Panels (a), (c), and (d) are adapted with permission from Springer Science and Business Media: Fenn, L. S.; McLean, J. A. Biomolecular structural separations by ion mobility-mass spectrometry. Anal. Bioanal. Chem. 2008, 391, 906. Panel (b) is adapted with permission from Springer Science and Business Media: Fenn, L. S. et al. Characterizing Ion Mobility-Mass Spectrometry Conformation Space for the Analysis of Complex Biological Samples. Anal. Bioanal. Chem. 2009, 394, 235.

The rapid structural separation of different molecular classes also provides a key advantage in omics measurements in that the separation of endogenous and exogenous material from the molecular class of interest is the separation of chemical noise. The separation of chemical noise provides two key benefits, for example in proteomics, namely: (1) eliminating database searching with signals that do not correspond to peptides, and (2) providing enhanced signal-to-noise ratios for low abundance peptides. By searching proteomic databases with only masses known to be putative peptides on the basis of structure, increases protein confidence level assignments by nearly a factor of 10 to 100 times over that using only integrated
MS data [32]. Furthermore, because IM-MS is a postionization technique, it can be combined with additional dimensions of LC separations to further differentiate analytes on the basis of additional physical characteristics such as hydrophobicity, etc. Clemmer and colleagues have recently described LC-ESI-IM-MS studies of the human plasma proteome, whereby 3D separations provided an enhanced concentration dynamic range, which is estimated to be $10^{5}$ to $10^{6}$ [33-35]. Owing to these advantages, IM-MS techniques are increasingly being used to study complex biological samples, such as whole cell lysates [36], plasma [33-35, 37], homogenized tissue [36, $38,39]$, or directly from thin tissue sections $[40,41]$. 


\section{Structural Separations in Conformation Space-A Correlation Fine Structure Examination}

The correlations of mobility with mass for a particular molecular class are logarithmic functions that capture the length squared verses length cubed relation of collision cross section with mass. Histogram plots of the appearance frequency of collision cross sections as the percent relative deviation from the average correlation reveal rather Gaussian shaped curves for large numbers of analytes for each biomolecular class [27]. However, hidden within these correlations is fine structure, which can provide additional information about the specific molecule. In the case of oligonucleotides, helical duplexes and G-quadruplexes have been shown to adopt larger structures than average globules [42-44], while single-stranded base stacking results in smaller than average structures [27]. For peptides, retained secondary structural elements can result in larger structures for $\alpha$-helices [45] and smaller structures for B-hairpins [46], respectively. Peptide structure is also affected in a predictable manner by post-translational modifications, such as phosphorylation $[47,48]$, glycosylation, cis-trans proline isomerization [49], disulfide bridging, and cyclization [50]. Carbohydrates that include a higher degree of branching than their linear counterparts adopt smaller structures, even when composed of the same individual carbohydrate monomers, and are isobaric species [51]. A summary of how different structural motifs and molecular sub-classes affect where these species are observed in relation to the average mobility-mass correlation is presented in Figure 3 for oligonucleotides, carbohydrates, peptides, lipids, and natural products.

The fine structure hidden within the nearly Gaussian histogram plots of the appearance frequency of collision cross sections as the percent relative deviation from the average correlation for a class can be rather dramatic as is the case for lipids. For example, deviations for lipids, as indicated in Figure 3, indicate that on average, sphingomyelins and cerebrosides adopt more extended structures, while phosphatidylcholines, phosphatidylethanolamines, and phosphatidylserines adopt more compact structures. Note that the two former and three latter classes of lipids belong to the larger families of sphingolipids and glycerophospholipids, respectively. Thus, the primary difference between the two families is connectivity of the fatty acyl tails to the phosphate and lipid head group via either a sphingosine or glycerol moiety. Although in a chemical connectivity sense this is not a large difference, this results in a bimodal distribution in a histogram plot of appearance fre-

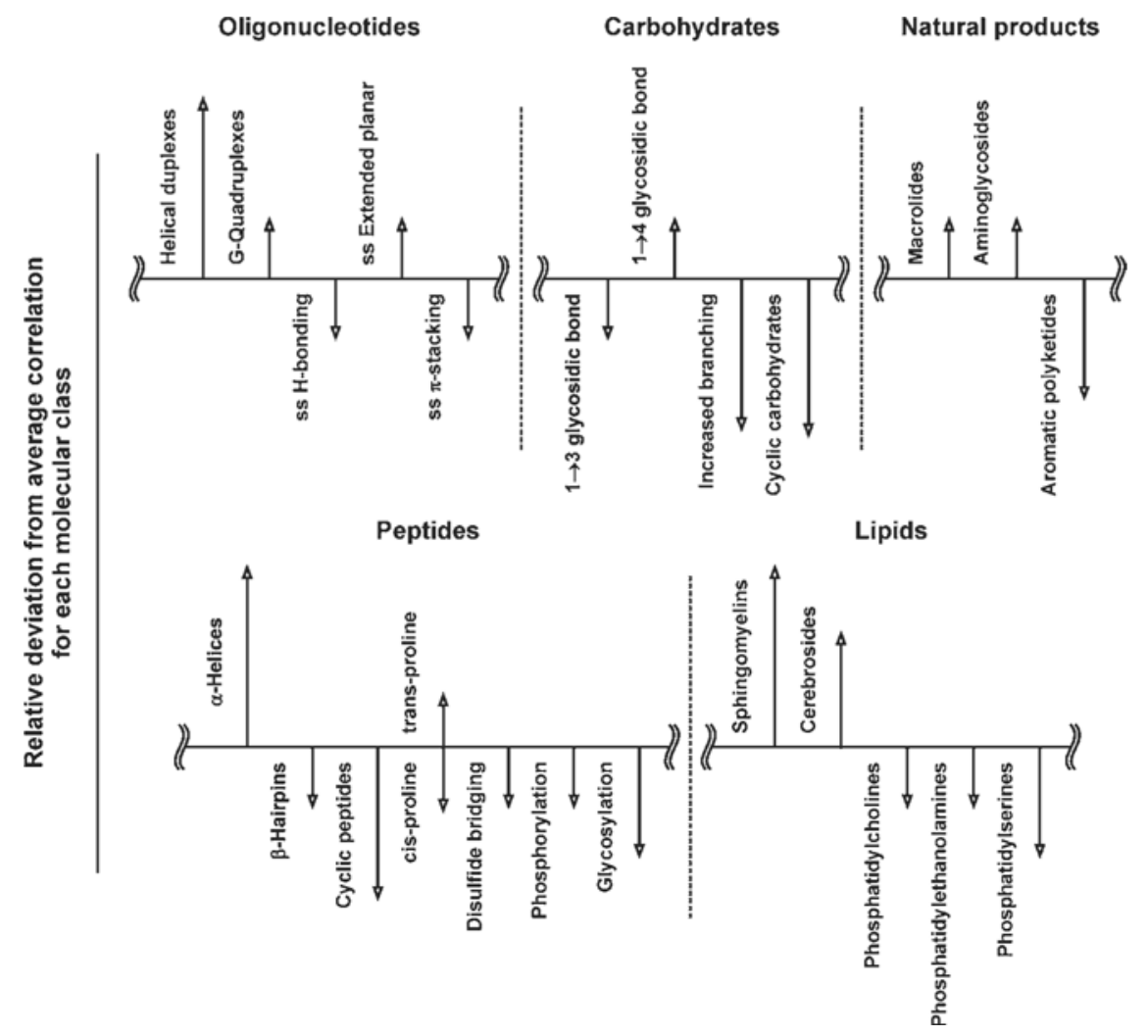

Figure 3. A plot depicting structural motifs and sub-classes of molecules that result in deviations from the average ion mobility $-\mathrm{m} / \mathrm{z}$ correlation for each of five different classes of biomolecules, including: oligonucleotides, carbohydrates, natural products, peptides, and lipids. The relative length and direction of individual arrows corresponds to the relative degree of deviation and whether those species are greater than or less than the predicted correlation for the average of molecules of that class. 
quency [52]. Thus, depending on the fine structural differences between sub-classes of biomolecules, the predicted appearance regions in conformation space is much more complex and richer in information than is commonly used.

Mapping these fine details should provide a means for better interpreting data from complex samples, and may provide a unique and high throughput means for screening and discovery. For example, bioactive secondary metabolite natural products in drug discovery are challenging to isolate and further to elucidate their structure. However, oftentimes this large class of molecules (e.g., cyclic peptides, macrolides, aminoglycosides, aromatic polyketides, etc.), exhibit broad functionality and are conformationally defined via multiple stereocenters, intramolecular cyclization, and incorporation of non-standard amino acids. Thus, the ability to map regions of conformation space corresponding to natural products may provide a guide-post to identification of new target compounds directly from crude extracts [50]. As the anhydrous structural landscape is further elucidated for biomolecules, both in a coarsegrained and fine structure perspective, the role of IM-MS methodologies in emerging bioanalytical and biophysical studies of complex biological samples will become increasingly prevalent. Ultimately, it is the coarse and fine structure in IM-MS correlations that gave rise to Horning's conclusion, and provide the unique information that the technique affords.

\section{Acknowledgments}

JAM thanks the many stimulating discussions with mentors, colleagues, collaborators, and S. Sundarapanian, L. Fenn, M. Kliman, T. J. Kerr, R. L. Gant-Branum, A. Mahsut, J. Enders, C. Goodwin, and W. Ridenour in the synthesis of the ideas presented here. Professor B. O. Bachmann (Department of Chemistry, VU) is thanked for the natural product library of compounds used and stimulating discussions. The author acknowledges financial support for this work by the Vanderbilt University College of Arts and Sciences, the Vanderbilt Institute of Chemical Biology, the American Society for Mass Spectrometry (Research Award), the Spectroscopy Society of Pittsburgh, and the Defense Threat Reduction Agency (grant no. HDTRA1-09-1-0013).

\section{References}

1. Röntgen, W. C. On a New Kind of Rays. Nature 1896, 53, 274-276.

2. Rutherford, E. Velocity and Rate of Recombination of the Ions of Gases Exposed to Röntgen Radiation. Phil. Mag. 1897, 44, 422-440.

3. Thomson, J. J. Rays of Positive Electricity. Proc. Royal Soc. 1913, A89, $1-20$.

4. Carr, T. W., Ed. Plasma Chromatography; Plenum Press: New York, 1984.

5. Bowers, M. T.; Kemper, P. R.; von Helden, G.; van Koppen, P. A. M. Gas-Phase Ion Chromatography: Transition Metal State Selection and Carbon Cluster Formation. Science 1993, 260, 1446-1451.

6. Borsdorf, H.; Eiceman, G. A. Ion Mobility Spectrometry: Principles and Applications. Appl. Spectrosc. Rev. 2006, 41, 323-375.

7. Eiceman, G. A.; Karpas, Z. Introduction to Ion Mobility Spectrometry. In: Ion Mobility Spectrometry, 2nd ed.; CRC Press: Boca Raton, 2005; pp 3-36.

8. Barnes, W. S.; Martin, D. W.; McDaniel, E. W. Mass Spectrographic Identification of the Ion Observed in Hydrogen Mobility Experiments. Phys. Rev. Lett. 1961, 6, 110-111.
9. McAfee, K. B. Jr.; Edelson, D. Identification and Mobility of Ions in a Townsend Discharge by Time-Resolved Mass Spectrometry. Proc. Phys. Soc. London. 1963, 81, 382-384.

10. Karasek, F. W.; Kilpatrick, W. D.; Cohen, M. J. Qualitative Studies of Trace Constituents by Plasma Chromatography. Anal. Chem. 1971, 43, 1441-1447.

11. Karasek, F. W.; Tatone, O. S. Plasma Chromatography of the Monohalogenated Benzenes. Anal. Chem. 1972, 44, 1758-1763.

12. Griffin, G. W.; Dzidic, I.; Carroll, D. I.; Stillwell, R. N.; Horning, E. C. Ion Mass Assignments Based on Mobility Measurements-Validity of Plasma Chromatographic Mass Mobility Correlations. Anal. Chem. 1973, 45, 1204-1209.

13. McDaniel, E. W.; Mason, E. A. The Mobility and Diffusion of Ions in Gases; John Wiley and Sons: New York, 1973.

14. Revercomb, H. E.; Mason, E. A. Theory of Plasma Chromatography/ Gaseous Electrophoresis-A Review. Anal. Chem. 1975, 47, 970-983.

15. Mason, E. A.; McDaniel, E. W. Transport Properties of Ions in Gases; John Wiley and Sons: New York, 1988

16. von Helden, G.; Wyttenbach, T.; Bowers, M. T. Conformation of Macromolecules in the Gas Phase: Use of Matrix-Assisted Laser Desorption Methods in Ion Chromatography. Science 1995, 267, 1483-1485.

17. Wyttenbach, T.; von Helden, G.; Bowers, M. T. Gas-Phase Conformation of Biological Molecules: Bradykinin. J. Am. Chem. Soc. 1996, 118 8355-8364.

18. Clemmer, D. E.; Hudgins, R. R.; Jarrold, M. F. Naked Protein Conformations: Cytochrome $c$ in the Gas Phase. J. Am. Chem. Soc. 1995, 117 10141-10142.

19. Shelimov, K. B.; Clemmer, D. E.; Hudgins, R. R.; Jarrold, M. F. Protein Structure in Vacuo: The Gas Phase Conformations of BPTI and Cytochrome c. I. Am. Chem. Soc. 1997, 119, 2240-2248.

20. Ruotolo, B. T.; Giles, K.; Campuzano, I.; Sandercock, A. M.; Bateman, R. H.; Robinson, C. V. Evidence for Macromolecular Rings in the Absence of Bulk Water. Science 2005, 310, 1658-1661.

21. Ruotolo, B. T.; Hyung, S.-J.; Robinson, P. M.; Giles, K.; Bateman, R. H. Robinson, C. V. Ion Mobility-Mass Spectrometry Reveals Long-Lived, Unfolded Intermediates in the Dissociation of Protein Complexes. Angew. Chem. Int. Ed. 2007, 46, 8001-8004.

22. Ruotolo, B. T.; Benesch, J. L. P.; Sandercock, A. M.; Hyung, S.-J.; Robinson, C. V. Ion Mobility-Mass Spectrometry Analysis of Large Protein Complexes. Nat. Protocols 2008, 3, 1139-1152.

23. Benesch, J. L. P. Collisional Activation of Protein Complexes: Picking Up the Pieces. J. Am. Soc. Mass Spectrom. 2009, 20, 341-348.

24. Sali, A.; Glaeser, R.; Earnest, T.; Baumeister, W. From Words to Literature in Structural Proteomics. Nature 2003, 422, 216-225.

25. Robinson, C. V.; Sali, A.; Baumeister, W. The Molecular Sociology of the Cell. Nature 2007, 450, 973-982.

26. Fenn, L. S.; McLean, J. A. Biomolecular Structural Separations by Ion Mobility-Mass Spectrometry. Anal. Bioanal. Chem. 2008, 391, 905-909.

27. Fenn, L. S.; Kliman, M.; Mahsut, A.; Zhao, S. R.; McLean, J. A. Characterizing Ion Mobility-Mass Spectrometry Conformation Space for the Analysis of Complex Biological Samples. Anal. Bioanal. Chem. 2009, 394, 235-244

28. Giddings, J. C. Two-Dimensional Separations: Concept and Promise. Anal. Chem. 1984, 56, 1258A-1270A.

29. McLean, J. A.; Ruotolo, B. T.; Gillig, K. J.; Russell, D. H. Ion MobilityMass Spectrometry: A New Paradigm for Proteomics. Int. J. Mass Spectrom. 2005, 240, 301-315.

30. Koomen, J. M.; Ruotolo, B. T.; Gillig, K. J.; McLean, J. A.; Russell, D. H.; Kang, M.; Dunbar, K. R.; Fuhrer, K.; Gonin, M.; Schultz, J. A. Oligonucleotide Analysis with MALDI-Ion Mobility-TOF-MS. Anal. Bioanal. Chem. 2002, 373, 612-617.

31. Woods, A. S.; Ugarov, M.; Egan, T.; Koomen, J.; Gillig, K. J.; Fuhrer, K. Gonin, M.; Schultz, J. A. Lipid/Peptide/Nucleotide Separation with MALDI-Ion Mobility-TOF-MS. Anal. Chem. 2004, 76, 2187-2195.

32. McLean, J. A.; Tao, L.; Perkins, S.; McLean, J. R.; Russell, D. H Multidimensional Proteomic Analysis of Escherichia coli. Whole Cell Lysates by HPLC-MALDI-Ion Mobility-MS: Extending Dynamic Range in Protein Analysis. In Proceedings of the 53rd ASMS Conference on Mass Spectrometry; Montreal, Canada, 2005.

33. Liu, X.; Plasencia, M.; Ragg, S.; Valentine, S. J.; Clemmer, D. E. Development of High Throughput Dispersive LC-Ion Mobility-TOF-MS Techniques for Analyzing the Human Plasma Proteome. Briefings Funct. Genomics Proteomics 2004, 3, 177-186.

34. Valentine, S. J.; Plasencia, M. D.; Liu, X.; Krishnan, M.; Naylor, S.; Udseth, H. R.; Smith, R. D.; Clemmer, D. E. Toward Plasma Proteome Profiling with Ion Mobility-Mass Spectrometry. J. Proteome Res. 2006, 5 , 2977-2984.

35. Liu, X.; Valentine, S. J.; Plasencia, M. D.; Trimpin, S.; Naylor, S.; Clemmer, D. E. Mapping the Human Plasma Proteome by SCX-LCIMS-MS. J. Am. Soc. Mass Spectrom. 2007, 18, 1249-1264.

36. Myung, S.; Lee, Y. J.; Moon, M. H.; Taraszka, J.; Sowell, R.; Koeniger, S. Hilderbrand, A. E.; Valentine, S. J.; Cherbas, L.; Cherbas, P.; Kaufmann, T. C.; Miller, D. F.; Mechref, Y.; Novotny, M. V.; Ewing, M. A.; Sporleder C. R.; Clemmer, D. E. Development of High-Sensitivity Ion Trap Ion Mobility Spectrometry Time-of-Flight Techniques: A High-Throughput Nano-LC-IMS-TOF Separation of Peptides Arising from a Drosophila Protein Extract. Anal. Chem. 2003, 75, 5137-5145.

37. Isailovic, D.; Kurulugama, R. T.; Plasencia, M. D.; Stokes, S. T. Kyselova, Z.; Goldman, R.; Mechref, Y.; Novotny, M. V.; Clemmer, D. E. 
Profiling of Human Serum Glycans Associated with Liver Cancer and Cirrhosis by IMS-MS. J. Proteome Res. 2008, 7, 1109-1117.

38. Liu, X.; Miller, B. R.; Rebec, G. V.; Clemmer, D. E. Protein Expression in the Striatum and Cortex Regions of the Brain for a Mouse Model of Huntington's Disease. J. Proteome Res. 2007, 6, 3134-3142.

39. Taraszka, J. A.; Kurulugama, R.; Sowell, R. A.; Valentine, S. J.; Koeniger, S. L.; Arnold, R. J.; Miller, D. F.; Kaufman, T. C.; Clemmer, D. E. Mapping the Proteome of Drosophila melanogaster: Analysis of Embryos and Adult Heads by LC-IMS-MS Methods. J. Proteome Res. 2005, 4, 1223-1237.

40. Jackson, S. N.; Ugarov, M.; Egan, T.; Post, J. D.; Langlais, D.; Schultz, J. A.; Woods, A. S. MALDI-Ion Mobility-TOF-MS Imaging of Lipids in Rat Brain Tissue. J. Mass Spectrom. 2007, 42, 1093-1098.

41. McLean, J. A.; Ridenour, W. B.; Caprioli, R. M. Profiling and Imaging of Tissues by Imaging Ion Mobility-Mass Spectrometry. J. Mass Spectrom. 2007, 42, 1099-1105.

42. Gidden, J.; Baker, E. S.; Ferzoco, A.; Bowers, M. T. Structural Motifs of DNA Complexes in the Gas Phase. Int. J. Mass Spectrom. 2005, 240, 183-193.

43. Baker, E. S.; Berstein, S. L.; Bowers, M. T. Structural Characterization of G-Quadruplexes in Deoxyguanosine Clusters Using Ion Mobility Mass Spectrometry. J. Am. Soc. Mass Spectrom. 2005, 16, 989-997.

44. Smargiasso, N.; Rosu, F.; Hsia, W.; Colson, P.; Baker, E. S.; Bowers, M. T.; De Pauw, E.; Gabelica, V. G. Quadruplex DNA Assemblies: Loop Length, Cation Identity, and Multimer Formation. J. Am. Chem. Soc. 2008, 130, 10208-10216.
45. Ruotolo, B. T.; Verbeck, G. F.; Thomson, L. M.; Gillig, K. J.; Russell, D. H. Observation of Conserved Solution-Phase Secondary Structure in GasPhase Tryptic Peptides. J. Am. Chem. Soc. 2002, 124, 4214-4215.

46. Wu, C.; Murray, M. M.; Bernstein, S. L.; Condron, M. M.; Bitan, G.; Shea, J.-E.; Bowers, M. T. The Structure of AB42 C-Terminal Fragments Probed by a Combined Experimental and Theoretical Study. J. Mol. Biol. 2009, 387, 492-501.

47. Ruotolo, B. T.; Verbeck, G. V.; Thomson, L. M.; Woods, A. S.; Gillig, K. J.; Russell, D. H. Distinguishing Between Phosphorylated and Nonphosphorylated Peptides with Ion Mobility-Mass Spectrometry. J. Proteome Res. 2002, 1, 303-306.

48. Thalassinos, K. Grabenauer, M.; Slade, S. E.; Hilton, G. R.; Bowers, M. T.; Scrivens, J. H. Characterization of Phosphorylated Peptides Using Traveling Wave-Based and Drift Cell Ion Mobility Mass Spectrometry. Anal. Chem. 2009, 81, 248-254.

49. Counterman, A. E.; Clemmer, D. E. Cis-trans Signatures of ProlineContaining Tryptic Peptides in the Gas Phase. Anal. Chem. 2002, 74, 1946-1951.

50. Fenn, L. S., Goodwin, C. R., Bachmann, B. O., McLean, J. A., unpublished. (Manuscript in preparation for J. Am. Chem. Soc. 2009.)

51. Fenn, L. S.; McLean, J. A. Enhanced Carbohydrate Structural Selectivity in Ion Mobility-Mass Spectrometry Analyses by Boronic Acid Derivatization. Chem. Commun. 2008, 43, 5505-5507.

52. Kliman, M.; Woods, A. S.; Schultz, J. A.; McLean, J. A. Fine Structure in Lipid Conformation Space: Study of Ion Mobility Mass Spectrometry Resolution of Sphingolipids and Glycerolipids. Proceedings of the 21st ASMS Conference; Sanibel, FL, 2009. 